MODELING, IDENTIFICATION AND CONTROL, 2001, VOL. 22, NO. 2, 89-101

doi:10.4173/mic.2001.23

\title{
Semi-Global Practical Stabilization and Disturbance Adaptation for an Underactuated Ship*
}

\author{
K. Y. PETTERSEN† and H. NIJMEIJER $\ddagger$ \\ Keywords: Nonlinear control, underactuated vehicles, disturbance adaptation, \\ dynamic positioning
}

\begin{abstract}
We consider the problem of stabilizing the position and orientation of a ship to constant desired values, when the ship has only two independent controls and also the ship is subject to an environmental force of unknown magnitude. We propose a time-varying feedback control law and a disturbance adaptation law, and show that this provides semi-global practical asymptotic stability. The control and adaptation laws are derived using a combined integrator backstepping and averaging approach. Simulation results are presented.
\end{abstract}

\section{Introduction}

Position and orientation control of ships are required in many offshore oil field operations, such as drilling, pipe-laying, tanking between ships, diving support, etc. The dynamic positioning (DP) control problem consists of finding a feedback control law that asymptotically stabilizes both the position and orientation to desired constant values. We consider the DP control problem for a ship that has no side thruster, but two independent main thrusters located at a distance from the centre line in order to provide both surge force and yaw moment. The control problem considered in this paper is to find a feedback control law that asymptotically stabilizes both the position variables and the orientation, using only the two available controls. As we attempt to control three degrees of freedom with only two independent controls, we have an underactuated control problem.

Control of underactuated ships is an active topic of research. Underactuated tracking control of ships has been considered for instance in Godhavn (1996), Fossen et al. (1998), Berge et al. (1998), Pettersen and Nijmeijer (1998), Sira-Ramirez (1999) and Bullo (2000). From results by Brockett (1983), Coron and Rosier (1994) and Zabczyk (1989) the ship is not stabilizable by static state feedback. The underactuated stabilization problem is thus not solvable using linear control theory or classical nonlinear control theory like static feedback linearization. In Wichlund et al. (1995) a continuous feedback control law is proposed that instead asymptotically stabilizes an equilibrium manifold. The desired equilibrium point is then stable as all the system variables are bounded by the initial conditions of the system. Furthermore, the position variables with this approach converge exponentially to their desired values. The course angle however converges to some constant value, but not

†Department of Engineering Cybernetics, Norwegian University of Science and Technology, N-7491 Trondheim, Norway.

$\ddagger$ Faculty of Mechanical Engineering, Eindhoven University of Technology, P.O. Box 513, 5600 MB Eindhoven, The Netherlands

*IEEE. Reprinted, with permission, from Proc. 39th IEEE Conference on Decision and Control, Sydney, Australia. 
necessarily to zero. In Reyhanoglu (1996) a discontinuous feedback control law is proposed, and this provides exponential convergence to the desired equilibrium point, under certain assumptions on the initial value. In Pettersen and Egeland (1996) a time-varying feedback control law is proposed that provides exponential stability of the desired equilibrium point. However the feedback law only locally stabilizes the desired equilibrium point. In Pettersen and Nijmeijer (1999) a time-varying feedback control law is proposed that provides semi-global practical exponential stability of a simplified model of the ship, where the surge and yaw velocities are considered as controls. In Fantoni et al. (2000) another simplified ship model is considered, a hovercraft, and based on passivity considerations and Lyapunov theory discontinuous control laws are proposed giving global convergence to the origin. In Bullo et al. (2000) a geometric framework for controllability analysis and motion control is proposed for mechanical systems on Lie groups, including the underactuated hovercraft. In this paper we propose a time-varying feedback control law that provides semi-global practical asymptotic stability of the ship dynamics with surge force and yaw moment controls.

Moreover, in previous works on underactuated ships and other underactuated vehicles, the influence of environmental disturbances has generally not been taken into account. Disturbances are included when considering the tracking control problem in Fossen et al. (1998) and Berge et al. (1998). For underactuated ship stabilization however, to the authors' best knowledge no results have been presented including disturbances in the control analysis and design. The experiments in Pettersen and Fossen (2000) showed that the ship with the locally exponentially stabilizing control law of Pettersen and Egeland (1996), experienced stationary oscillations. Simulations indicated that the main reason for the oscillatory behavior was the environmental disturbances. It was thus pointed out that a topic of future research should be the inclusion of a constant environmental force in the analysis and control design, possibly including adaptation in the control scheme, in order to reduce or eliminate the stationary errors and oscillations, which is the motivation for this paper. With the approach developed in this paper, we still have stationary oscillations, but we are able to affect the amplitude of the oscillations. We consider here the DP problem when the ship is subject to an environmental disturbance, i.e. stabilizing a constant position and orientation while compensating for a disturbance of unknown magnitude giving force components in both surge and sway and also a yaw moment, when only surge force and yaw moment controls are available. To this end we propose a time-varying feedback control law and an adaptation law that provide semi-global practical asymptotic stability. The paper is organized as follows. In Section II the model of the ship is presented. In Section III the control and adaptation laws are developed and proved to semi-globally practically stabilize the underactuated ship. Simulation results are presented in Section IV.

\section{The ship model}

We use the following ship model Fossen (1994)

$$
\begin{gathered}
M \dot{v}+C(v) v+D v=\tau+F_{\text {ext }} \\
\dot{\eta}=J(\eta) v
\end{gathered}
$$

where $v=[u, v, r]^{T}$ are the linear velocities in surge (body-fixed $x$-direction) and sway (body-fixed $y$-direction) and angular velocity in yaw (about body-fixed 
$z$-axis) respectively. The configuration vector $\eta=[x, y, \psi]^{T}$ denotes the position and orientation of the ship in earth-fixed coordinates. The matrix $M$ is the matrix of inertia, including added mass effects. It has the properties $(d / d t) M=0$ and $s^{T} M s>0 \forall s \in \mathbb{R}^{3}$. The $D$ matrix represents the hydrodynamic damping and has the properties $(d / d t) D=0$ and $s^{T} D s>0 \forall s \in \mathbb{R}^{3}$. We assume that the matrices $M$ and $D$ are diagonal, i.e.

$$
\begin{gathered}
M=\operatorname{diag}\left\{m_{11}, m_{22}, m_{33}\right\} \\
D=\operatorname{diag}\left\{d_{11}, d_{22}, d_{33}\right\}
\end{gathered}
$$

This is true for ships having port/starboard and fore/aft symmetry, when the axes of the body-fixed coordinate system are chosen parallel to the principal axes of the displaced fluid, which are equal to the principal axes of the ship. Most ships have port/starboard symmetry. Non-symmetry fore/aft of the ship implies that $m_{23}=m_{32} \neq 0$ and $d_{23} \neq d_{32} \neq 0$. These terms will, however, be small compared to the diagonal elements $m_{i i}$ and $d_{i i}(i=1 \ldots 3)$ for most ships. Control design under a relaxation of this assumption to the general case is trivial to solve for fully actuated ships, while it is a topic for future research for underactuated ships.

The Coriolis and centripetal matrix is

$$
C(v)=\left[\begin{array}{ccc}
0 & 0 & -m_{22} v \\
0 & 0 & m_{11} u \\
m_{22} v & -m_{11} u & 0
\end{array}\right]
$$

and we note that it is skew-symmetric.

The $J$ matrix represents the kinematic transformation from body-fixed to Earthfixed coordinates

$$
J(\eta)=\left[\begin{array}{ccc}
\cos (\psi) & -\sin (\psi) & 0 \\
\sin (\psi) & \cos (\psi) & 0 \\
0 & 0 & 1
\end{array}\right]
$$

The ship is subject to environmental disturbances like wind, waves and ocean currents. We do not want the ship to react to the high-frequent components of the environmental disturbances, as this would only give increased wear and tear on the thrusters. Instead we want the control system to compensate for the low-pass filtered bias of these disturbances. The disturbance is thus modeled as a constant or slowlyvarying force. The angle between the disturbance force and the earth-fixed $x$-axes is denoted by $\gamma$. We assume that the direction of the force can be measured, but the magnitude of the environmental force, $F$, is not measurable, and we will thus have to estimate this. We will control the rotation of the ship around a desired centre of rotation (CR) where the origin of the body-fixed coordinate system is placed. This will be located at the center line, but not necessarily coincide with the center of gravity (CG). The distance between CG and CR along the center line we denote by $a$. The forces and moment due to environmental disturbances are then given by

$$
F_{e x t}=\left[\begin{array}{c}
F \cos (\psi-\gamma) \\
-F \sin (\psi-\gamma) \\
-F a \sin (1 /-\gamma)
\end{array}\right]
$$


The vector of controls is

$$
\tau=\left[\begin{array}{c}
\tau_{u} \\
0 \\
\tau_{r}
\end{array}\right]
$$

\section{Semi-global practical stabilization}

The dynamic positioning control problem considered in this section, is to find a feedback law that asymptotically stabilizes the origin $(\eta, v)=(0,0)$ when the ship is subject to an environmental disturbance of arbitrary direction given by $\gamma$, and of a bounded but unknown magnitude $F$. Note that compensating for the sway component of $F_{\text {ext }}$ would be significantly simplified if the ship was fully actuated, such that $\tau_{v} \neq 0$.

The dynamic equations of the ship can be rewritten as

$$
\begin{aligned}
M \dot{v}+\bar{C}(v) v+D v & =\left[\begin{array}{c}
\tau_{u}+F \cos (\psi-\gamma) \\
-m_{11} u r-F \sin (\psi-\gamma) \\
\tau_{r}+m_{11} u v-F a \sin (\psi-\gamma)
\end{array}\right] \\
\dot{\eta} & =J(\eta) v
\end{aligned}
$$

where

$$
\bar{C}(v)=\left[\begin{array}{ccc}
0 & 0 & -m_{22} v \\
0 & 0 & 0 \\
m_{22} v & 0 & 0
\end{array}\right]
$$

is a skew-symmetric matrix. For the development of the control law, we will consider the term $m_{11} u r$ as the input to the second of the three dynamic equations in (9). Define

$$
\begin{gathered}
u_{d}=\frac{1}{\sqrt{m_{11}}} \rho \sin (\omega t) \\
r_{d}=-\frac{2}{\sqrt{m_{11}} \rho}\left(-d_{c 2} v+k_{x} \sin (\psi) x-k_{y} \cos (\psi) y+\hat{F} \sin (\psi-\gamma)\right) \sin (\omega t)
\end{gathered}
$$

where

$$
\rho(x, y, \psi, v)=\alpha \sqrt{\sin ^{2}(\psi-\gamma)+x^{2}+y^{2}+\psi^{2}+v^{2}+\varepsilon^{2}}
$$

and $\alpha, \varepsilon, d_{c 2}, k_{x}, k_{y}, \omega>0$ are constant control parameters, and where $\hat{F}$ is the disturbance estimate given by

$$
\hat{F}=\frac{1}{\sigma}(\cos (\psi-\gamma) u-\sin (\psi-\gamma) v-a \sin (\psi-\gamma)(r+\lambda \psi))
$$

where the constant parameters $\sigma, \lambda>0$. We define $\tilde{F}=\hat{F}-F$. 
Let $v_{d}=\left[u_{d}, 0, r_{d}\right]^{T}$. We define the new coordinates

$$
\begin{aligned}
& \bar{u}=u-u_{d} \\
& \bar{v}=v \\
& \bar{r}=r-r_{d}
\end{aligned}
$$

and $\bar{v}=[\bar{u}, \bar{v}, \bar{r}]^{T}$. The system equations in the new coordinates are

$$
\begin{gathered}
M \dot{\bar{v}}+\bar{C}\left(\bar{v}+v_{d}\right)+D\left(\bar{v}+v_{d}\right)= \\
{\left[\begin{array}{c}
\tau_{u}+F \cos (\psi-\gamma) \\
-m_{11}\left(\bar{u} \bar{r}+\bar{u} r_{d}+\bar{r} u_{d}+u_{d} r_{d}\right)-F \sin (\psi-\gamma) \\
\tau_{r}+m_{11}\left(\bar{u}+u_{d}\right) \nu-F a \sin (\psi-\gamma)
\end{array}\right]-M \dot{v}_{d}} \\
\dot{\eta}=J(\eta)\left(\bar{v}+v_{d}\right) \\
\hat{F}=\frac{1}{\sigma}\left(\cos (\psi-\gamma)\left(\bar{u}+u_{d}\right)-\sin (\psi-\gamma) \bar{v}-a \sin (\psi-\gamma)\left(\bar{r}+r_{d}+\lambda \psi\right)\right)
\end{gathered}
$$

Based on recursive vector-backstepping controller design, we propose the following control law

$$
\begin{gathered}
\tau_{u}=-\hat{F} \cos (\psi-\gamma)-d_{c 1} \bar{u}-k_{x} \cos (\psi) x-k_{y} \sin (\psi) y-\lambda\left(m_{11}-m_{22}\right) \psi \bar{v} \\
\tau_{r}=\hat{F} a \sin (\psi-\gamma)-d_{c 3} \bar{r}-k_{\psi} \psi-\lambda m_{33} \bar{r}-\lambda d_{33} \psi
\end{gathered}
$$

where the constant control parameters $d_{c 1}, d_{c 2}, d_{c 3}, k_{x}, k_{y}, k_{\psi}, \lambda>0$.

The averaged system equations of the closed loop system (19-23), are then (Sanders and Verhulst, 1985)

$$
\begin{gathered}
M \bar{v}+\tilde{C}(\bar{v}) \bar{v}+D \bar{v}= \\
{\left[\begin{array}{c}
-\tilde{F} \cos (\psi-\gamma)-d_{c 1} \bar{u}-k_{x} \cos (\psi) x-k_{y} \sin (\psi) y-\lambda\left(m_{11}-m_{22}\right) \psi \bar{v} \\
-m_{11} \bar{u} \bar{r}-d_{c 2} \bar{v}+k_{x} \sin (\psi) x-k_{y} \cos (\psi) y+\tilde{F} \sin (\psi-\gamma) \\
m_{11} \bar{u} \bar{v}+\tilde{F} a \sin (\psi-\gamma)-d_{c 3} \bar{r}-k_{\psi} \psi-\lambda m_{33} \bar{r}-\lambda d_{33} \psi
\end{array}\right]} \\
\dot{\eta}=J(\eta) \bar{v} \\
\hat{F}=\frac{1}{\sigma}(\cos (\psi-\gamma) \bar{u}-\sin (\psi-\gamma) \bar{v}-a \sin (\psi-\gamma)(\bar{r}+\lambda \psi))
\end{gathered}
$$

For this system we choose the Lyapunov function candidate

$$
V=\frac{1}{2}(\bar{D}+\Lambda \eta)^{T} M(\bar{v}+\Lambda \eta)+\frac{1}{2} \eta^{T} K \eta+\frac{1}{2} \sigma \tilde{F}^{2}
$$

where

$$
\Lambda=\left[\begin{array}{lll}
0 & 0 & 0 \\
0 & 0 & 0 \\
0 & 0 & \lambda
\end{array}\right]
$$




$$
K=\left[\begin{array}{ccc}
k_{x} & 0 & 0 \\
0 & k_{y} & 0 \\
0 & 0 & k_{\psi}
\end{array}\right]
$$

The time derivative of $V$ along the averaged system vector field is

$$
\begin{aligned}
\dot{V}= & (\bar{v}+\Lambda \eta)^{T}(M \Lambda \dot{\eta}+\bar{C}(\bar{v}) \Lambda \eta-D(\bar{v}+\Lambda \eta)+D \Lambda \eta \\
& \left.+\left[\begin{array}{c}
-\tilde{F} \cos (\psi-\gamma)-d_{c 1} \bar{u}-k_{x} \cos (\psi) x-k_{y} \sin (\psi) y-\lambda\left(m_{11}-m_{22}\right) \psi \bar{v} \\
-m_{11} \bar{u} \bar{r}-d_{c 2} \bar{v}+k_{x} \sin (\psi) x-k_{y} \cos (\psi) y+\tilde{F} \sin (\psi-\gamma) \\
m_{11} \bar{u} \bar{v}+\tilde{F} a \sin (\psi-\gamma)-d_{c 3} \bar{r}-k_{\psi} \psi-\lambda m_{33} \bar{r}-\lambda d_{33} \psi
\end{array}\right]\right) \\
& +\eta^{T} K \dot{\eta}+\sigma \tilde{F} \hat{F}
\end{aligned}
$$

A simple manipulation of the equations yields

$$
\begin{gathered}
\dot{V}=(\bar{v}+\Lambda \eta)^{T}(M \Lambda \dot{\eta}+\bar{C}(\bar{v}) \Lambda \eta-D(\bar{v}+\Lambda \eta)+D \Lambda \eta \\
\left.+\left[\begin{array}{c}
\lambda m_{11} \psi \bar{v}-\tilde{F} \cos (\psi-\gamma)-d_{c 1} \bar{u}-k_{x} \cos (\psi) x-k_{y} \sin (\psi) y-\lambda\left(m_{11}-m_{22}\right) \psi \bar{v} \\
-d_{c 2} \bar{v}+k_{x} \sin (\psi) x-k_{y} \cos (\psi) y+\tilde{F} \sin (\psi-\gamma) \\
\tilde{F} a \sin (\psi-\gamma)-d_{c 3} \bar{r}-k_{\psi} \psi-\lambda m_{33} \bar{r}-\lambda d_{33} \psi
\end{array}\right]\right) \\
+\eta^{T} K \dot{\eta}+\sigma \tilde{F} \hat{F} \\
=(\bar{v}+\Lambda \eta)^{T}\left(-D(\bar{v}+\Lambda \eta)+\tilde{F}\left[\begin{array}{c}
-\cos (\psi-\gamma) \\
\sin (\psi-\gamma) \\
a \sin (\psi-\gamma)
\end{array}\right]\right) \\
\left.\quad-\left[\begin{array}{ccc}
d_{c 1} & 0 \\
0 & d_{c 2} & 0 \\
0 & 0 & d_{c 3}
\end{array}\right](\bar{v}+\Lambda \eta)-J^{T} K \eta\right)+\eta^{T} K J \bar{v}+\sigma \tilde{F} \hat{F}
\end{gathered}
$$

Define

$$
D^{c}=\left[\begin{array}{ccc}
d_{c 1} & 0 & 0 \\
0 & d_{c 2} & 0 \\
0 & 0 & d_{c 3}
\end{array}\right]
$$

Using the adaptation law (15) we get

$$
\dot{V}=-(\bar{v}+\Lambda \eta)^{T}\left(D+D^{c}\right)(\bar{v}+\Lambda \eta)-\lambda k_{\psi} \psi^{2}
$$

Thus $\dot{V}$ is negative semi-definite. As the averaged system is autonomous, we can use La Salle's theorem. The set $R=\{(\bar{v}, \eta, \tilde{F}) \mid \dot{V}=0\}=\{(\bar{v}, \eta, \tilde{F}) \mid(\bar{v}=0, \psi=0\}$.

$$
\left.M \dot{\bar{\nu}}\right|_{R}=-K\left[\begin{array}{l}
x \\
y \\
0
\end{array}\right]+\tilde{F}\left[\begin{array}{c}
-\cos (\gamma) \\
-\sin (\gamma) \\
-a \sin (\gamma)
\end{array}\right]
$$


If $a \neq 0$, the last of these three equations implies that unless the desired direction of the ship is exactly parallel to the direction of the disturbance (i.e. unless $\sin \gamma=0$ ), then for any solution that stays in $R$ we have that $\tilde{F}=0$, and thus by the first and second equation also $x$ and $y$ have to be zero as $K$ is a non-singular matrix. So in this case the whole state vector converges to zero.

We have thus proved the following result

Proposition 1: If $a \sin (\gamma) \neq 0$, then the origin $(\bar{v}, \eta, \tilde{F})=(0,0,0)$ of the averaged closed-loop system (24-26) is globally asymptotically stable.

Then, by Teel et al. (1999) we have the following proposition. Note that $\omega$ is the frequency of the time-varying functions (12-13)

Proposition 2: If $a \sin (\gamma) \neq 0$, then there exists a constant $\omega^{*}>0$ such that for $\omega^{*}<\omega<\infty$ the origin of the closed-loop system (19-23) is semi-globally practically asymptotically stable.

Therefore, if the desired center of rotation CR is not placed at the center of gravity, i.e. a $a \neq 0$, and the desired direction of the ship is not chosen parallel to the direction of the environmental disturbance, i.e. $\sin (\gamma) \neq 0$, then with suffciently high frequency of the time-varying control the ship converges to an arbitrarily small neighborhood of the desired configuration.

\section{Simulation results}

The ship model used in the simulations describes Cybership I which is a model of an offshore supply vessel scale 1:70, having a mass of $17.6 \mathrm{~kg}$ and a length of $1.2 \mathrm{~m}$. The desired CR where the origin of the body-fixed coordinate system is placed, is located $40 \mathrm{~cm}$ in front of the CG along the body-fixed $x$-axis, hence $a=-0.4 \mathrm{~m}$. The magnitude of the environmental disturbance was assumed to be $F=0.07 \mathrm{~N}$, which is similar to the environmental disturbance experienced at the Guidance and Navigation Laboratory, NTNU, for this ship during experiments reported in Pettersen and Fossen (2000). In these experiments the locally stabilizing time-varying feedback control law from Pettersen and Egeland (1996), with integral action but without disturbance adaptation, is used for the control of the model ship.

The experiments in Pettersen and Fossen (2000) showed that the ship with the locally exponentially stabilizing control law experienced continual oscillations about stationary errors. The oscillations could be due to a combination of several factors, like modelling errors, actuator saturation, measurement noise and environmental disturbances like currents and waves. However, simulations indicated that the main reason for the oscillatory behavior was the environmental disturbances. It was thus pointed out as a topic of future research to include a constant environmental force in the analysis and control design, possibly including adaptation in the control scheme, in order to reduce or eliminate the stationary errors and oscillations, which was the motivation for the work presented in this paper. With the approach developed in this paper, we will still have stationary oscillations, but we are able to affect the amplitude of the oscillations. From (12-13) we see that when the state variables $x, y$, $\psi, \bar{u}, \bar{v}, \bar{r}$ and $\tilde{F}$ have converged to zero, the amplitude of the oscillations in $u$ and $r$ will be approximately

$$
u \approx \frac{\alpha}{\sqrt{m_{11}}} \sin (\gamma) \sin (\omega t)
$$




$$
r \approx-\frac{2}{\sqrt{m_{11} \alpha}} F \sin (\omega t)
$$

assuming that $\varepsilon<<\sin \gamma$. We note that the smaller $\sin (\gamma)$ is, the smaller the amplitude of the stationary oscillations in $u$ will be. In practice one typically wants to head the ship quite close to the opposite of the direction of the environmental force, in order to decrease the sway force and yaw moment resulting from the environmental force. From Proposition 1 we see that we cannot choose $\sin (\gamma)=0$ (since we need some excitation for all the system variables to converge). We will therefore have some oscillations in $u$. Also note, that the larger the magnitude of the disturbance force is, the higher is the amplitude of the oscillations in $r$. The scaling factor $\alpha$ is a tool for increasing/reducing the amplitude of the oscillations in $u$ in proportion to $r$.

The $\gamma$ value of the simulation was $\gamma=0.087 \mathrm{rad}=5 \mathrm{deg}$. No apriori information about $F$ was assumed. Guided by linear control theory applied to the linearization of the averaged system (24-26) about the origin, we chose the following control parameters

$$
\begin{aligned}
& k_{x}=10 d_{c 1}=150 \\
& k_{y}=1 d_{c 2}=40 \\
& k_{\psi}=0.5 d_{c 3}=15 \\
& \lambda=1 \sigma=10 \\
& \varepsilon=0.001 \omega=0.5 \\
& \alpha=0.8
\end{aligned}
$$

Initially the ship was at rest, with a deviation of $-2[\mathrm{~m}]$ in the $x$-direction and -1 $[\mathrm{m}]$ in the $y$-direction from the desired position at the origin. Figure 1 shows the trajectory of the ship in the $x y$-plane, and Figures 2-9 the time evolution of the state variables and controls. The simulations showed that the ship converged to a small neighborhood of the origin. Comparing the simulation results to the experimental results presented in Pettersen and Fossen (2000) the simulations indicated that the control law proposed in this paper demands more high-frequent control in order to

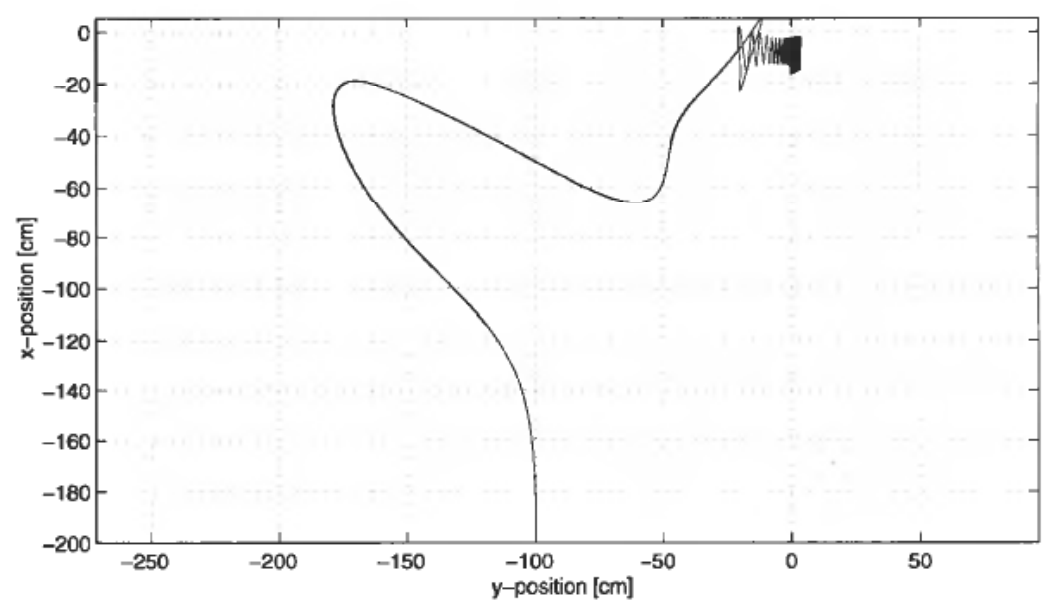

Figure 1. The trajectory in the $x y$-plane. 


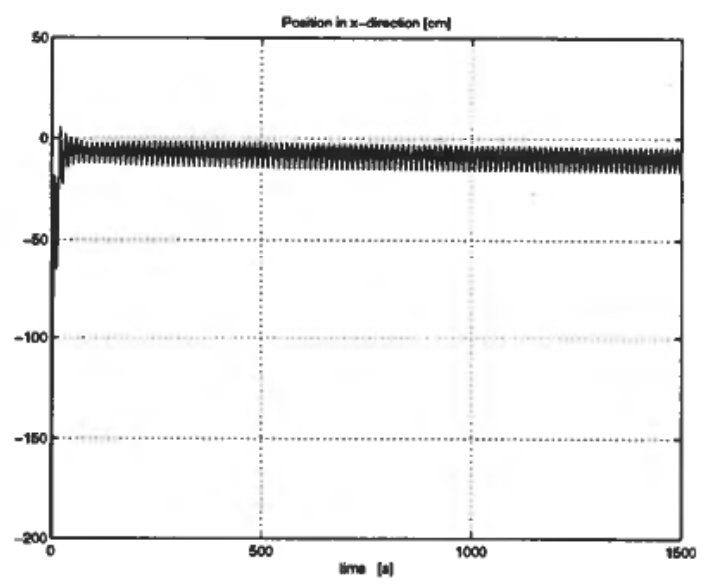

Figure 2. The position variable $x[\mathrm{~cm}]$.

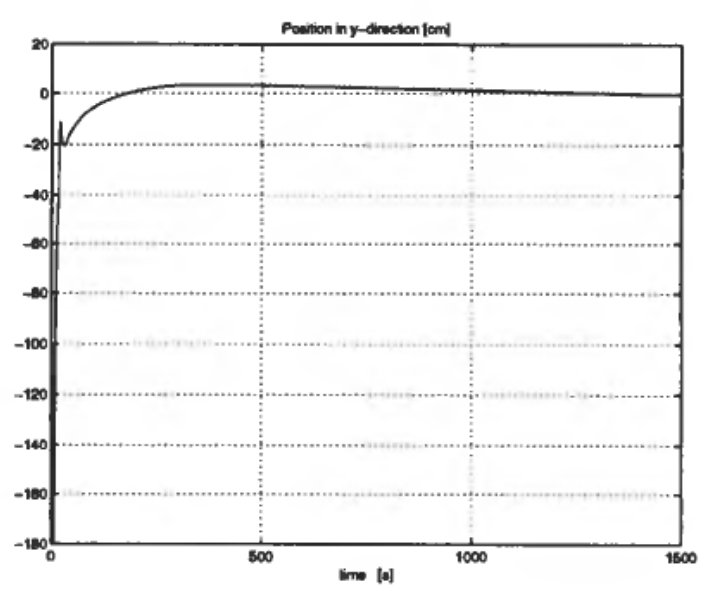

Figure 3. The position variable $y[\mathrm{~cm}]$.

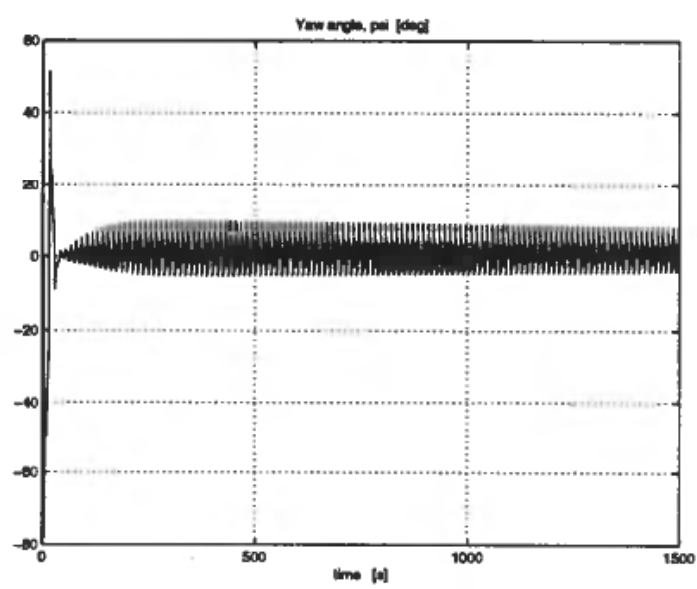

Figure 4. The orientation $\psi$ [deg]. 


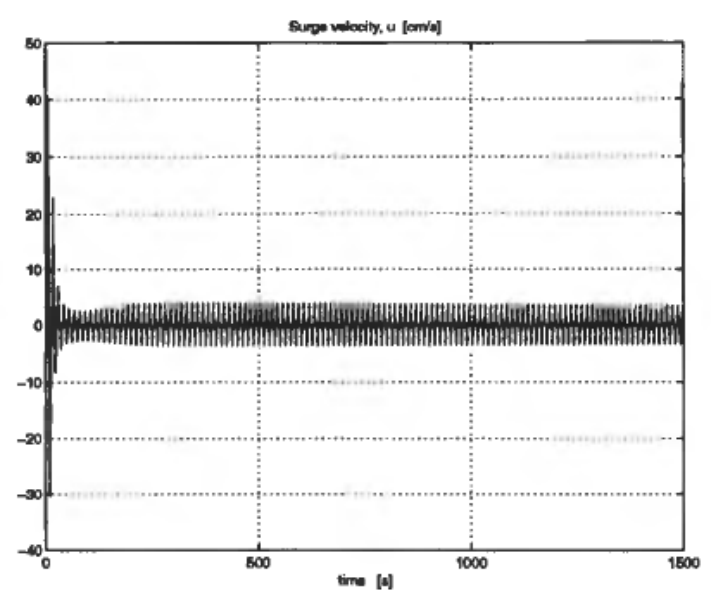

Figure 5. The surge velocity $u[\mathrm{~cm} / \mathrm{s}]$.

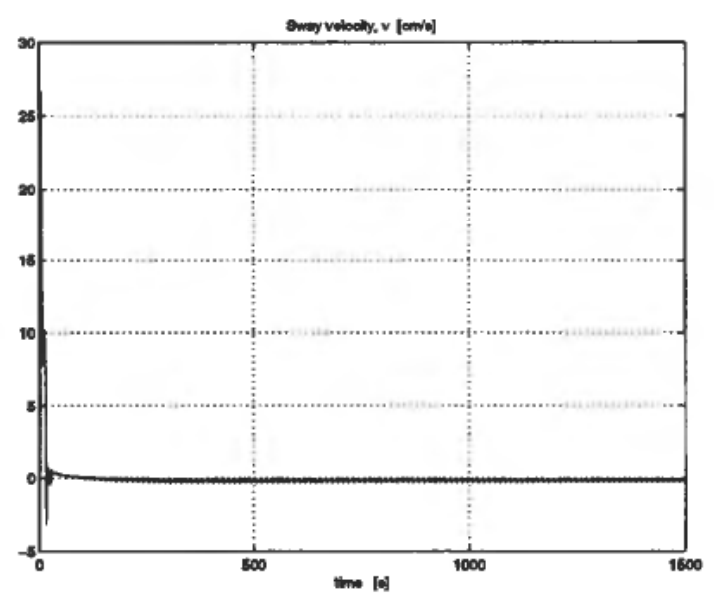

Figure 6. The sway velocity $v[\mathrm{~cm} / \mathrm{s}]$.

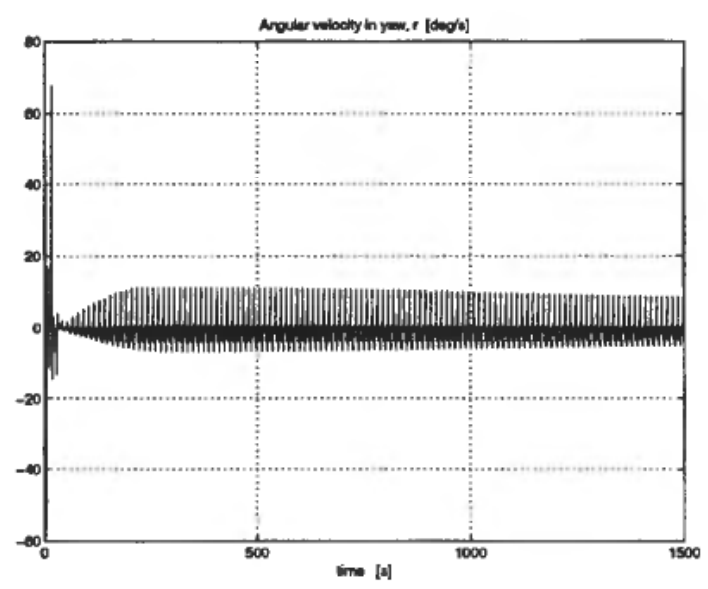

Figure 7. The yaw velocity $r[\mathrm{deg} / \mathrm{s}]$. 


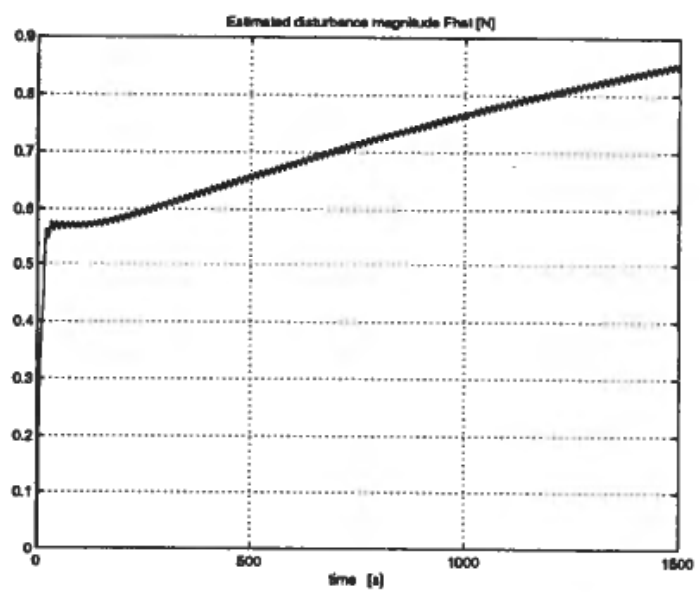

Figure 8. The disturbance estimate $\hat{F}[\mathrm{~N}]$.

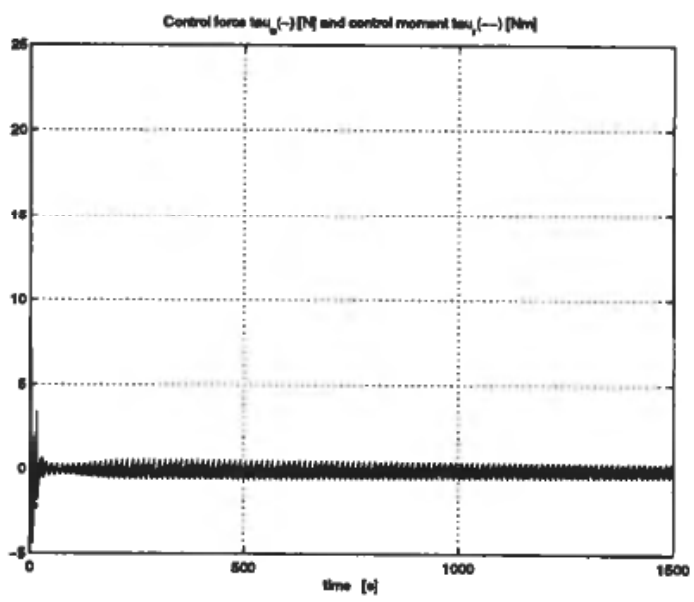

Figure 9. The force and moment controls $\tau_{u}[\mathrm{~N}]$ and $\tau_{r}[\mathrm{Nm}]$.

give a stable scheme. How close to the origin the ship converged, depended strongly on the control parameters and also on the direction of the environmental force, $\gamma$.

We note that the adaptation error converged quite slowly, as the adaptation parameter was chosen quite large in order for the eigenvalue of the $\tilde{F}$-dynamics (for the linearized averaged system) to be well below the bandwidth of the other system variables, and also as smaller values of $\sigma$ were seen to give larger stationary errors in $y$.

\section{Conclusion}

Few results have been reported on the control of underactuated systems in the presence of environmental disturbances. In Pettersen and Fossen (2000) experiments were performed with the time-varying controller proposed in Pettersen and Egeland (1996) extended with integral action, for the dynamic positioning of an underactuated ship. While simulations with the proposed control law showed exponential convergence to the desired position and orientation, the experiments revealed that the ship 
would instead oscillate around stationary errors. Further simulations indicated that the main reason for this was a constant environmental force acting on the ship, and this motivated the work presented in this paper, where a constant environmental force was included in the analysis and control design.

In the paper the dynamic positioning problem was considered when the underactuated ship was subject to a disturbance of unknown magnitude giving force components in both surge and sway together with a yaw moment, when only surge force and yaw moment controls were available. To this end, we proposed a timevarying feedback control law and an adaptation law. This gave semi-global practical asymptotic stability. Simulation results were presented and these showed how the ship converged to a neighborhood of the desired position and orientation. With the approach developed in this paper, there will still be stationary oscillations, but we are able to affect the amplitude of the oscillations. The simulations indicated that the control law proposed in this paper compared to Pettersen and Fossen (2000) demands more high-frequent control in order to give a stable scheme. Also, how close the ship converged to the origin depended strongly on the control parameters and on the direction of the environmental force. Furthermore, the convergence of the disturbance estimate was quite slow. The simulations thus indicated that the controller has several drawbacks in practical applications, but the approach can hopefully motivate future research on how to take environmental disturbances into account for the control of underactuated systems.

\section{REFERENCES}

BERGE, S. P., OHTSU, K. and Fossen, T. I. (1998). Nonlinear control of ships minimizing the position tracking errors. In Proc. of the IFAC Conference on Control Appl. in Marine Syst., pp. 141-147, Fukuoka, Japan.

BROCKETT, R. W. (1983). Asymptotic stability and feedback stabilization. In R. W. BROCKETT, R. S. Millman, and H. J. Sussmann, editors, Differential Geometric Control Theory, pp. 181-191. Birkhäuser.

BulLo, F. Stabilization of relative equilibria for underactuated systems on riemannian manifolds. Automatica, Dec. 2000, vol 36:12, pp. 1819-1834.

Bullo, F., Leonard, N. E. and Lewis, A. D. (1999). Controllability and motion algorithms for underactuated lagrangian systems on lie groups. IEEE Transactions on Automatic Control, Aug. 2000, vol 45:8, pp. 1437-1454.

CORON, J.-M. and Rosier, L. (1994). A relation between continuous time-varying and discontinuous feedback stabilization. J. of Math. Syst. Estimation, and Control, 4(1):67-84.

Fantoni, I., Lozano, R., Mazenc, F. and Pettersen, K. Y. (2000). Stabilization of a nonlinear underactuated hovercraft. International Journal of Robust and Nonlinear Control, 10(8): 645-654.

Fossen, T. I. (1994). Guidance and Control of Ocean Vehicles. John Wiley \& Sons Ltd, Chichester.

Fossen, T. I., Godhavn, J.-M., Berge, S. P. and LindegaARD, K.-P. (1998). Nonlinear control of underactuated ships with forward speed compensation. In Proc. of IFAC NOLCOS98, pp. 121-127, Enschede, The Netherlands.

GodHAVN, J.-M. (1996). Nonlinear tracking of underactuated surface vessels. In Proc. 35th IEEE Conf. on Decision and Control, pp. 987-991, Kobe, Japan.

PetTersen, K. Y. and Egeland, O. (1996). Exponential stabilization of an underactuated surface vessel. In Proc. 35th IEEE Conf. on Decision and Control, pp. 967-971, Kobe, Japan.

Pettersen, K. Y. and Fossen, T. I. (2000). Underactuated dynamic positioning of a shipexperimental results. IEEE Transactions on Control Systems Technology, vol 8, no. 5, pp. 856-863.

Pettersen, K. Y. and Numeijer, H. (1998). Tracking control of an underactuated surface 
vessel. In Proc. 37th IEEE Conf. on Decision and Control, pp. 4561-4566, Tampa, Florida.

Pettersen, K. Y. and NiJmeiJer, H. (1999). Global practical stabilization and tracking for an underactuated ship a combined averaging and backstepping approach. Modeling, Identication and Control, 20(4):189-199.

ReyHanoglu, M. (1996). Control and stabilization of an underactuated surface vessel. In Proc. 35th IEEE Conf. on Decision and Control, pp. 2371-2376, Kobe, Japan.

SANDERS, J. A. and Verhulst, F. (1985). Averaging Methods in Nonlinear Dynamical Systems. Springer-Verlag, New York.

SiRA-RAmireZ, H. (1999). On the control of the underactuated ship: A trajectory planning approach. In Proc. 38th IEEE Conf. on Decision and Control, pp. 1658-1663, Phoenix, AZ.

Teel, A. R., Peuteman, J. and Aeyels, D. (1999). Semi-global practical asymptotic stability and averaging. Systems and Control Letters, 37(5):329-334.

Wichlund, K. Y., Sørdalen, O. J. and Egeland, O. (1995). Control of vehicles with secondorder nonholonomic constraints: Underactuated vehicles. In Proc. Third Eur. Control Conf., pp. 3086-3091, Rome, Italy.

ZABCZYK, J. (1989). Some comments on stabilizability. Appl. Math. and Optimization, 19:1-9. 